\title{
Correction to: Cloning and Expression of Heterologous Cellulases and Enzymes in Aspergillus niger
}

\author{
Morgann C. Reilly, Saori Amaike Campen, Blake A. Simmons, \\ Scott E. Baker, John M. Gladden, and Jon K. Magnuson
}

Correction to:

Chapter 10 in: Mette Lübeck (ed.), Cellulases: Methods and Protocols, Methods in Molecular Biology, vol. 1796, https://doi.org/10.1007/978-1-4939-7877-9_10

The author's family name were incorrectly published in the original version. This has been corrected to read as:

GivenName: Saori

FamilyName: Amaike Campen 